\title{
Composição de espécies vasculares de campo sujo e campo úmido em área de cerrado, Itirapina - SP, Brasil ${ }^{1}$
}

\author{
JOÃO L.S. TANNUS ${ }^{2}$ e MARCO ANTONIO ASSIS ${ }^{2,3}$
}

(recebido: 4 de junho de 2003; aceito: 1 de abril de 2004)

\begin{abstract}
Vascular species composition of dry and swamp grasslands in a savanna area, Itirapina - SP, Brazil) Floristic composition of an area of savanna (cerrado) at Itirapina region, São Paulo State (22 $155^{\prime} 43^{\prime}$ ' -50' ' S and 47 $53^{\prime}$ - 54' W), presenting dry and swamp grasslands (campo sujo and campo úmido), was studied and compared to distinguish this physiognomies. Collects were carried out monthly from March 1998 to April 1999, and sporadically from May 1999 to December 2001. In addition, a survey of previously collected plants of this area was conducted at the Herbário Rioclarense (HRCB) collection. The results were analysed according to the presence of such species in the physiognomies and to the floristic components. A total of 384 species, 211 genera and 76 families of vascular plants were sampled. 255 out of the total were exclusively found in the dry grassland, 114 were found only in swamp grassland. Only 10 species were present in both physiognomies. The richest families found in the dry grassland were: Asteraceae, Leguminosae and Poaceae. Among the richest families found in the swamp grassland Cyperaceae, Poaceae, Lentibulariaceae and Eriocaulaceae were the most important. The proportion between herbaceous and woody floras of dry grassland was 3.6:1. No woody species were found in swamp grassland. This study showed that floristic distinction between dry and swamp grassland is pronounced due to the small number of species these areas share.
\end{abstract}

Key words - Brazilian vegetation, floristic composition, herbaceous swamp, tropical grasslands, wetlands

RESUMO - (Composição de espécies vasculares de campo sujo e campo úmido em área de cerrado, Itirapina - SP, Brasil). Foi estudada a composição florística de uma área de cerrado na região de Itirapina - SP (22 15 ' 43 ' '-50' 'S e 47 53 '- 54' W), onde ocorrem fisionomias de campo sujo e campo úmido. Visando estabelecer uma melhor distinção entre estas fisionomias, foi realizado o levantamento florístico que incluiu coletas em excursões mensais no período de março de 1998 a abril de 1999 , além de coletas esporádicas a partir de maio de 1999 até dezembro de 2001. Adicionalmente foi realizado um levantamento junto ao Herbário Rioclarense (HRCB) de todas as coletas provenientes desta área. Os resultados foram analisados em função da ocorrência das espécies nas fisionomias (campo sujo e campo úmido), e por componente florístico (herbáceo-subarbustivo e arbustivo-arbóreo). Foram amostradas 384 espécies, 211 gêneros e 76 famílias de plantas vasculares. Do total de espécies, 255 são exclusivas do campo sujo e 114 do campo úmido. Apenas 10 espécies tiveram ocorrência comum entre as duas fisionomias. Dentre as famílias mais ricas do campo sujo destacam-se: Asteraceae, Leguminosae e Poaceae. No campo úmido, as famílias mais ricas são Cyperaceae, Poaceae, Lentibulariaceae e Eriocaulaceae. No campo sujo o componente herbáceo-subarbustivo prevaleceu sobre o arbustivo-arbóreo numa proporção de 3,6:1. No campo úmido foram encontradas apenas espécies herbáceosubarbustivas. $\mathrm{O}$ estudo revelou acentuadas distinções florísticas entre o campo sujo e o campo úmido, tendo em vista o reduzido número de espécies compartilhadas.

Palavras-chave - campo sujo, campo úmido, cerrado, composição florística, Itirapina

\section{Introdução}

As vegetações próprias do cerrado (sensu lato) apresentam variações fisionômico-florísticas consideráveis, desde formas florestais densas até campos puramente herbáceos (Coutinho 1978, Eiten 1983, 1992, Klink et al. 1993). Em decorrência dessa gama de variação estrutural e florística, o cerrado (s.l.)

\footnotetext{
1. Parte da dissertação de mestrado do primeiro autor

2. Universidade Estadual Paulista, Instituto de Biociências, Departamento de Botânica, Caixa Postal 199, 13506-900 Rio Claro, SP, Brasil.

3. Autor para correspondência: massis@ rc.unesp.br
}

tem sido tratado como um complexo vegetacional (Coutinho 1978, Rizzini 1979, Eiten 1983). Segundo o conceito "floresta - ecótono - campo" (Coutinho 1978), o cerrado (s.l.) é composto por duas floras distintas, sendo uma puramente arbustivo-arbórea, predominante nas fisionomias florestais (cerradão), e outra herbáceosubarbustiva, característica das fisionomias campestres (campo limpo). As fisionomias savânicas, ou seja, as formas intermediárias ou ecótonos (cerrado sensu stricto, campo cerrado e campo sujo), caracterizam-se pela ocorrência de uma flora mista, composta por elementos florestais e campestres (Coutinho 1978, 2002).

Do ponto de vista da diversidade, estudos recentes demonstram que a riqueza florística do cerrado (s.l.) 
pode superar, em muito, o que inicialmente se supunha (Castro et al. 1999). Uma das constatações mais evidentes é que a maioria dos estudos florísticos foi realizada com base em levantamentos fitossociológicos, levando-se em conta, principalmente, espécies arbustivoarbóreas (Mantovani \& Martins 1993). Se por um lado a flora arbustivo-arbórea do cerrado (s.l.) é relativamente bem estudada, por outro, o reduzido número de levantamentos da flora herbáceosubarbustiva restringe seu conhecimento a poucas localidades (Ratter et al. 1997, Castro et al. 1999).

Considerando-se que o cerrado (s.l.) é constituído, em sua maioria, por fisionomias savânicas, sua maior riqueza florística pode se encontrar no componente não arbóreo, ou seja, na flora herbáceo-subarbustiva (Castro et al. 1999). Estimativas pouco precisas da riqueza dessa flora têm sugerido cerca de 2.000 a 5.000 espécies (Ratter et al. 1997, Mendonça et al. 1998, Castro et al. 1999). Entretanto, diante da escassez de estudos para esse componente florístico, os dados são pouco conclusivos (Ratter et al. 1997, Castro et al. 1999, Filgueiras 2002). Adicionalmente, estudos sobre o componente herbáceo-subarbustivo indicam a ocorrência de grandes mudanças na sua composição entre diferentes regiões, demonstrando tratar-se de uma flora sensível a variações de clima, solo, e intensidade de queimadas, entre outros fatores (Loefgren 1890, Mantovani \& Martins 1993, Filgueiras 2002).

Além das fitocenoses consideradas próprias do cerrado (s.l.) (cerradão, cerrado s.str., campo cerrado, campo sujo e campo limpo), outras, tais como as matas de galeria, matas mesófilas, veredas e campos úmidos, ocorrem freqüentemente no contexto do bioma Cerrado, porém de forma menos expressiva (Rizzini 1979, Oliveira Filho et al. 1989, Eiten 1992, Ratter et al. 1997, Pivello et al. 1998). Entre essas formações, os campos úmidos se traduzem como fisionomias campestres, de caráter estritamente herbáceo-subarbustivo, que se estabelecem em solos temporária ou permanentemente encharcados, devido ao afloramento do lençol freático associado à deficiência de drenagem (Eiten 1963, 1992, Couto et al. 1985, Schiavini \& Araújo 1989, Ratter et al. 1997, Pivello et al. 1998, Ribeiro \& Walter 1998, Araújo et al. 2002). Com relativa frequiência, os campos úmidos são encontrados como encraves em áreas do Cerrado, ocupando os fundos de vales, ou associados a cursos d'água (Oliveira Filho et al. 1989, Schiavini \& Araújo 1989, Eiten 1992, Ratter et al. 1997). Ainda que possa haver grande número de coletas botânicas provenientes de campos úmidos, as quais muitas vezes são incluídas em listagens da flora do bioma Cerrado (e.g., Mendonça et al. 1998), estudos florísticos detalhados destas formações são disponíveis apenas para algumas localidades (Araújo et al. 2002, Guimarães et al. 2002). Grande parte dos trabalhos que tratam dos campos úmidos se refere a estudos de solos (Couto et al. 1985), ou trazem abordagens sobre a fisionomia e estrutura da vegetação (Ribeiro et al. 1983, Schiavini \& Araújo 1989).

No presente trabalho, estudou-se a composição da flora vascular de uma área de ocorrência de campo sujo e campo úmido na região de Itirapina (SP), visando reconhecer, comparar e estabelecer uma melhor distinção entre estas fisionomias, contribuindo para o conhecimento da flora do cerrado, sobretudo do componente herbáceo-subarbustivo, bem como do campo úmido para o qual existem poucos trabalhos disponíveis.

\section{Material e métodos}

A área de estudo abrange aproximadamente 65 ha e localiza-se numa propriedade particular denominada Fazenda da Máquina, no município de Itirapina, SP (figura 1), tendo um de seus limites junto à rodovia SP-225 (Brotas-Itirapina), à altura do km 107 (22¹5’43”-50”' S e 4753'-54’ W), a uma altitude aproximada de 700 metros. Nesta área, assim como no restante da fazenda, verifica-se uma vegetação remanescente de cerrado que tem sido utilizada para a pecuária do tipo extensiva. Devido à pressão exercida pelo pastoreio, a vegetação nativa encontra-se fortemente antropizada. Além do pisoteio do gado, constata-se a presença de pequenas manchas da espécie exótica Brachiaria decumbens, e as queimadas anuais são freqüentes.

Segundo o sistema de classificação de Köppen (1948), o clima regional é do tipo Cwa "mesotérmico com inverno seco e verão chuvoso" (Prado 1997). Durante o período de 1980 a 2001, as médias mensais das temperaturas mínimas e máximas registradas foram $17^{\circ} \mathrm{Ce} 23,5^{\circ} \mathrm{C}$. A precipitação anual média foi de $1.523 \mathrm{~mm}$, com duas estações bem definidas: chuvosa de outubro a março (1.258 $\mathrm{mm})$, e seca de abril a setembro (265 $\mathrm{mm}$ ) (dados da Estação Climatológica do CRHEA/EESC- USP, localizada a cerca de $15 \mathrm{~km}$ da área de estudo).

Quanto à geomorfologia, a região encontra-se inserida na unidade denominada "Província de Cuestas Basálticas", no Planalto de São Carlos, situada um pouco além das escarpas que estabelecem os limites com a Depressão Periférica, ou seja, no reverso das Cuestas (Ponçano et al. 1981). A topografia local é relativamente plana, com ligeiras depressões de baixas amplitudes onde o lençol freático aflora durante a estação chuvosa e parte da estação seca. De acordo com Prado (1997), o solo predominante na área é do tipo "Areia quartzosa álica A moderado", sendo muito permeável e de baixo potencial nutricional. Entretanto, nos locais de afloramento do lençol freático o solo é essencialmente orgânico. 


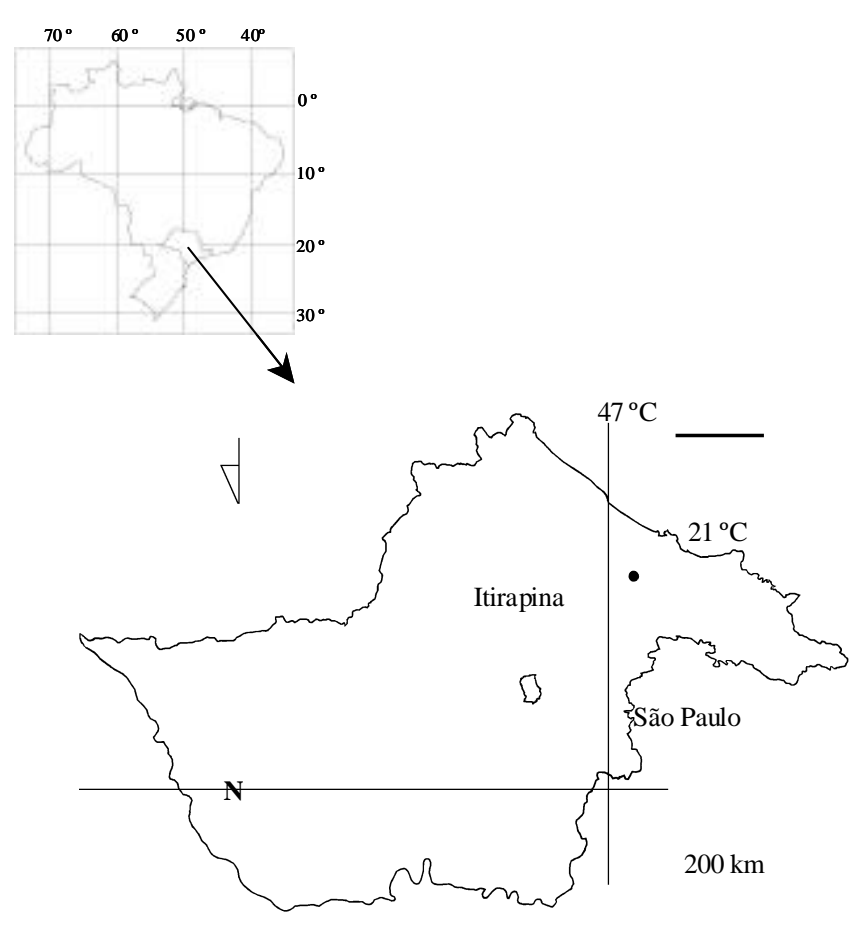

Figura 1. Localização do município de Itirapina-SP, Brasil.

Figure 1. Localization of the municipality of Itirapina-SP, Brazil.

A cobertura vegetal da área caracteriza-se pela ocorrência de duas fisionomias que se estabelecem de acordo com as características fisiográficas do terreno, as quais, por sua vez, resultam em diferentes condições de drenagem. Nas porções mais elevadas, sobre solos bem drenados, ocorre a fisionomia de campo sujo (senso Coutinho 1978), que recobre dois terços da área de estudo e predomina em toda a extensão da fazenda (figura 2). Restrita às depressões úmidas, onde o terreno é periodicamente alagado, encontra-se a fisionomia de campo úmido (senso Ribeiro \& Walter 1998), sendo, portanto, menos conspícua em comparação ao campo sujo.
As coletas de material botânico foram realizadas durante um dia de cada mês, no período de março de 1998 a abril de 1999, quando não houve registros de queimadas e, esporadicamente, até dezembro de 2001, quando houveram ao menos duas queimadas parciais da área. Em cada excursão foram feitas caminhadas aleatórias ao longo de toda a área de estudo (65 ha), no intuito de amostrar todas as espécies vasculares em estágio reprodutivo e registrar a ocorrência das mesmas nas diferentes fisionomias.

Os materiais coletados foram herborizados e incluídos no Herbário do Instituto de Biociências da Universidade Estadual Paulista - Campus de Rio Claro (HRCB). As identificações foram feitas por meio de bibliografia especializada, tendo como base a Flora Brasiliensis de Martius, por comparação com materiais do HRCB e com auxílio de especialistas. Adicionalmente, foi realizado um levantamento junto ao Herbário (HRCB) de todo material coletado anteriormente nesta mesma área, procedente de outros trabalhos vinculados ao Departamento de Botânica da Universidade Estadual Paulista - Campus de Rio Claro. Neste caso, as identificações foram revistas. Para as angiospermas seguiu-se o sistema de classificação de Cronquist (1988), excetuando-se as Leguminosae, abordadas como única família (senso Polhill \& Raven 1981). Para as pteridófitas adotou-se o sistema de Tryon \& Tryon (1982).

As espécies foram classificadas quanto às formas de vida de acordo com o sistema de Raunkiaer (1934), adaptado por Mueller-Dombois \& Ellenberg (1974) sendo que as caméfitas, geófitas, hemicriptófitas, lianas, parasitas e terófitas foram consideradas como pertencentes ao componente herbáceo-subarbustivo, enquanto as fanerófitas foram consideradas como arbustivo-arbóreas. No caso das espécies encontradas na coleção do HRCB, a indicação das formas de vida foi feita com base nos dados de etiqueta, através de consulta à literatura, e das próprias observações de campo.

Espécies tidas como ruderais foram apontadas de acordo com Lorenzi (1991), Kissman \& Groth (1992, 1995), Kissman (1997) e Mendonça et al. (1998).

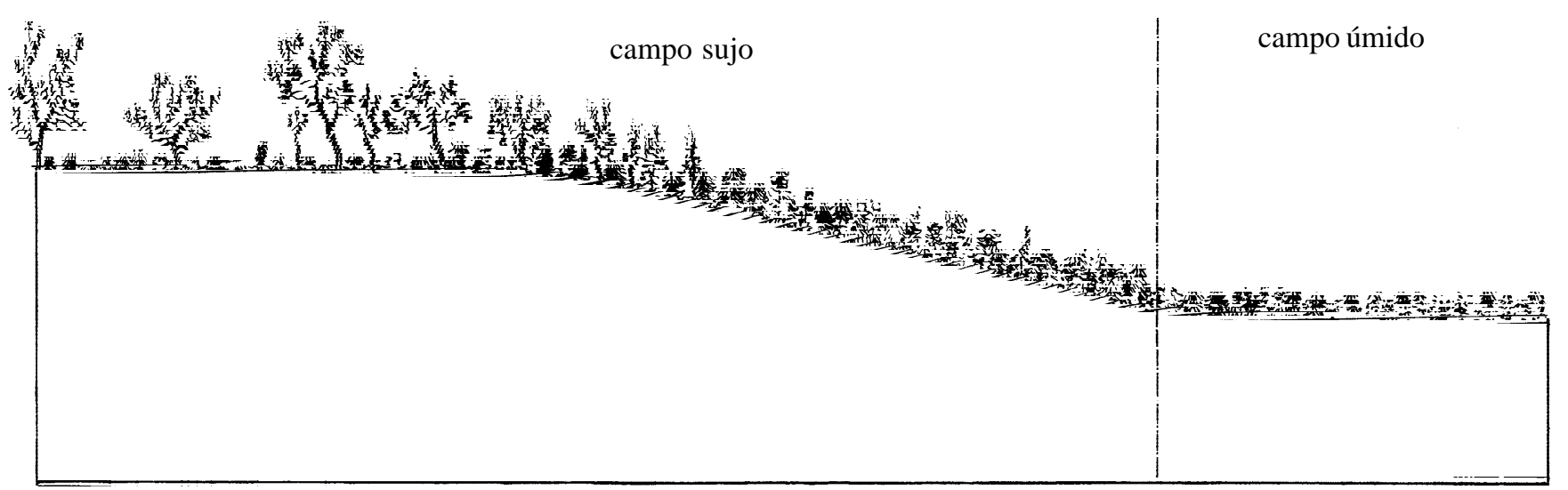

Figura 2. Diagrama perfil da vegetação da área estudada.

Figure 2. Vegetation profile diagram of the studied area. 
Os resultados foram analisados em função da ocorrência das espécies nas fisionomias (campo sujo e campo úmido), e por componente florístico (herbáceo-subarbustivo e arbustivo-arbóreo). As espécies levantadas na coleção do HRCB foram atribuídas para o campo sujo ou campo úmido de acordo com os dados de etiqueta.

\section{Resultados}

Foram amostradas 381 espécies de angiospermas, distribuídas em 209 gêneros e 74 famílias, além de três espécies de pteridófitas (tabela 1). A maioria das espécies $(75 \%)$ provém de coletas realizadas no período de 1998 a 1999; poucas foram acrescidas pelas coletas posteriores (apenas 12). Entretanto, o levantamento retroativo da coleção do Herbário HRCB contribuiu com
84 espécies, $22 \%$ da flora local, as quais não foram encontradas no campo, em estádio reprodutivo, durante o período deste estudo. Do total de espécies listadas, $60(15,6 \%)$ são consideradas ruderais (tabela 1$)$.

$\mathrm{Na}$ área de estudo a fisionomia de campo sujo caracteriza-se pela cobertura herbácea densa, principalmente graminosa, sobre a qual ocorrem indivíduos subarbustivos, arbustivos e, com menor freqüência, indivíduos arbóreos de pequeno porte (até 3 $\mathrm{m}$ de altura). Já a fisionomia de campo úmido apresenta cobertura essencialmente graminosa, com subarbustos muito esparsos.

Com relação a essas diferentes fisionomias, $66,4 \%$ das espécies levantadas foram registradas exclusivamente no campo sujo e $29,7 \%$ somente no campo úmido (tabela 1). As espécies compartilhadas

Tabela 1. Lista das espécies vasculares coletadas na área estudada em Itirapina, SP $\left(22^{\circ} 15^{\prime} 43^{\prime \prime}-50^{\prime \prime} \mathrm{S}\right.$ e $\left.47^{\circ} 53^{\prime}-54^{\prime} \mathrm{W}\right)$. C.S. = campo sujo, C.U. = campo úmido; $\mathrm{ND}=$ hábitat não definido; F.V. = forma de vida (Cam: caméfita, Fan: fanerófita, Geo: geófita, Hem: hemicriptófita, Li: liana, Pr: parasita, Ter: terófita); $\mathrm{HRCB}=$ número de registro no HRCB.

Table 1. Checklist of vascular plant species of studied area in Itirapina, SP $\left(22^{\circ} 15^{\prime} 43^{\prime \prime}-50^{\prime \prime} \mathrm{S}\right.$ and $\left.47^{\circ} 53^{\prime}-54^{\prime} \mathrm{W}\right)$. C.S. = dry grassland, C.U. = swamp grassland; ND = habitat not defined; F.V. = Life forms (Cam: chamaephytes, Fan: phanerophytes, Geo: geophytes, Hem: hemicryptophytes, Li: vines, Pr: parasites, Ter: terophytes); HRCB = voucher number at HRCB.

\begin{tabular}{|c|c|c|c|}
\hline Família/Espécie & F.V. & Hábitat & HRCB \\
\hline \multicolumn{4}{|l|}{ ACANTHACEAE } \\
\hline Ruellia bulbifera Lindau & Geo & C.S. & 28567 \\
\hline Ruellia sp.* & Geo & C.S. & 27418 \\
\hline \multicolumn{4}{|l|}{ ALISMATACEAE } \\
\hline Echinodorus paniculatus Micheli* & Geo & C.U. & 3836 \\
\hline \multicolumn{4}{|l|}{ AMARANTHACEAE } \\
\hline Froelichia procera (Seub.) Pedersen & Geo & C.S. & 28047 \\
\hline Gomphrena agrestis Mart.* & Geo & C.S. & 24670 \\
\hline Gomphrena graminea Moq. & Geo & C.S. & 29991 \\
\hline Gomphrena macrocephala A. St.-Hil. & Hem & C.S. & 29773 \\
\hline Gomphrena virgata Mart.* & Geo & C.S. & 8208 \\
\hline Pfaffia helichrysoides (Mart.) Kuntze & Geo & C.S. & 28553 \\
\hline Pfaffia jubata Moq.* & Geo & C.S. & 26999 \\
\hline \multicolumn{4}{|l|}{ AMARYLLIDACEAE } \\
\hline Zephyranthes sp.* & Geo & C.U. & 5922 \\
\hline \multicolumn{4}{|l|}{ ANACARDIACEAE } \\
\hline Anacardium humile A. St.-Hil.* & Cam & C.S. & 4482 \\
\hline \multicolumn{4}{|l|}{ ANNONACEAE } \\
\hline Annona coriacea Mart. & Fan & C.S. & 29774 \\
\hline Annona crassiflora Mart. & Fan & C.S. & 29740 \\
\hline Annona sp. & Cam & C.S. & 29402 \\
\hline Duguetia furfuracea (A. St.-Hil.) Benth. \& Hook. f. & Fan & C.S. & 28165 \\
\hline \multicolumn{4}{|l|}{ APIACEAE } \\
\hline Eryngium ebracteatum Lam. & Hem & C.U. & 29381 \\
\hline Eryngium juncifolium (Urb.) Mathias \& Constance & Hem & C.S. & 29749 \\
\hline
\end{tabular}


continuação

\begin{tabular}{|c|c|c|c|}
\hline Família/Espécie & F.V. & Hábitat & $\mathrm{HRCB}$ \\
\hline \multicolumn{4}{|l|}{ APOCYNACEAE } \\
\hline Aspidosperma tomentosum Mart.* & Fan & C.S. & 4515 \\
\hline Aspidosperma sp.* & Fan & C.S. & 8166 \\
\hline Hancornia speciosa Gomez & Fan & C.S. & 29739 \\
\hline Himatanthus obovatus (Müll. Arg.) Woodson var. obovatus * & Fan & C.S. & 5045 \\
\hline Macrosiphonia virescens (A. St-Hil.) Müll. Arg. & Hem & C.S. & 29411 \\
\hline Mandevilla illustris (Vell.) Woodson & Hem & C.S. & 29738 \\
\hline Mandevilla pohliana (Stadelm.) A.H. Gentry & Hem & C.S. & 29735 \\
\hline Rhabdadenia pohlii Müll. Arg. & $\mathrm{Li}$ & C.U. & 29694 \\
\hline \multicolumn{4}{|l|}{ ARACEAE } \\
\hline Urospatha aff. edwalli Engl. & Geo & C.U. & 30041 \\
\hline Xanthosoma striatipes (Kunth) Madison* & Geo & C.U. & 3839 \\
\hline \multicolumn{4}{|l|}{ ARECACEAE } \\
\hline Syagrus cf. microphylla Burret & Cam & C.S. & 28050 \\
\hline \multicolumn{4}{|l|}{ ASCLEPIADACEAE } \\
\hline Blepharodon bicuspidatum E. Fourn. & $\mathrm{Li}$ & C.S. & 35151 \\
\hline Hemipogon acerosus Decne. & Hem & C.S. & 29747 \\
\hline Oxypetalum aequaliflorum E. Fourn. & Ter & C.S. & 29366 \\
\hline Oxypetalum marginatum Malme & Ter & C.S. & 29405 \\
\hline Oxypetalum martii E. Fourn. f. paulista Hoehne & Ter & C.S. & 29369 \\
\hline Oxypetalum pachygynum Decne. & $\mathrm{Li}$ & C.S./C.U. & 30006 \\
\hline \multicolumn{4}{|l|}{ ASTERACEAE } \\
\hline Acanthospermum australe (Loefl.) Kuntze $\mathrm{e}^{1,2}$ & Ter & C.S. & 28542 \\
\hline Achyrocline alata (Kunth) DC. & Ter & C.S./C.U. & 29379 \\
\hline Aspilia reflexa Baker & Geo & C.S. & 29744 \\
\hline Aspilia setosa Griseb. & Geo & C.S. & 29414 \\
\hline Baccharis coridifolia DC. ${ }^{1,2}$ & Hem & C.S. & 30322 \\
\hline Baccharis dracunculifolia DC. ${ }^{1,2}$ & Cam & C.S. & 28157 \\
\hline Baccharis illinita DC.* & Cam & C.S. & 3856 \\
\hline Baccharis trimera (Less.) DC. ${ }^{1,2}$ & Hem & C.U. & 38604 \\
\hline Baccharis vismioides DC.* & Hem & C.U. & 8164 \\
\hline Baccharis sp.* & Hem & C.S. & 27003 \\
\hline Bidens gardneri Baker & Ter & C.S. & 30033 \\
\hline Calea cuneifolia DC. & Hem & C.S. & 29359 \\
\hline Calea uniflora Less. & Hem & C.S. & 28126 \\
\hline Chaptalia integerrima (Vell.) Burkart ${ }^{1,2}$ & Hem & C.S. & 29150 \\
\hline Chresta sphaerocephala DC.* & Cam & C.S. & 7466 \\
\hline Conyza bonariensis (L.) Cronquist $\mathrm{t}^{1,2}$ & Ter & C.S. & 30080 \\
\hline Elephantopus biflorus (Less.) Sch. Bip. & Hem & C.S. & 28508 \\
\hline Emilia sonchifolia $\left(\right.$ L.) DC. ${ }^{1}$ & Ter & C.S. & 28148 \\
\hline Erechtites hieracifolia (L.) Rafin. ex DC.*1 & Ter & C.S. & 7464 \\
\hline Eupatorium betonicaeforme (DC.) Baker & Hem & C.U. & 29993 \\
\hline Eupatorium bupleurifolium var. linifolia Baker* & Hem & C.S. & 4620 \\
\hline Eupatorium campestre DC. & Hem & C.S. & 29397 \\
\hline Eupatorium congestum Hook. \& Arn.* & Hem & C.S. & 5600 \\
\hline Eupatorium kleinioides Kunth & Hem & C.S. & 29992 \\
\hline Eupatorium oxylepis DC. & Cam & C.S. & 28523 \\
\hline Eupatorium pauciflorum Kunth & Ter & C.S. & 28048 \\
\hline Eupatorium ramosissimum Gardner & Hem & C.S. & 30027 \\
\hline Eupatorium squalidum DC.*1,2 & Ter & C.S. & 5568 \\
\hline Eupatorium vauthierianum DC. & Cam & C.S. & 28941 \\
\hline Eupatorium sp. & Hem & C.S. & 30538 \\
\hline
\end{tabular}


continuação

\begin{tabular}{|c|c|c|c|}
\hline Família/Espécie & F.V. & Hábitat & $\mathrm{HRCB}$ \\
\hline \multicolumn{4}{|l|}{ ASTERACEAE (cont.) } \\
\hline Gamochaeta americana (Mill.) Wedd. ${ }^{1}$ & Ter & C.S. & 28946 \\
\hline Gochnatia pulchra Cabrera & Cam & C.S. & 28152 \\
\hline Gochnatia velutina (Bong.) Cabrera & Cam & C.S. & 28942 \\
\hline Hoehnephyton trixioides (Gardner) Cabrera & Hem & C.S. & 28579 \\
\hline Isostigma megapotamicum (Spreng.) Blake & Hem & C.S. & 29761 \\
\hline Jaegeria hirta (Lag.) Less. & Ter & C.S. & 30051 \\
\hline Leucopsis tweediei Baker* & Hem & C.U. & 3838 \\
\hline Mikania micrantha Kunth & $\mathrm{Li}$ & C.S./C.U. & 28151 \\
\hline Mikania sp.* & Hem & C.S. & 24691 \\
\hline Piptocarpha rotundifolia (Less.) Baker & Fan & C.S. & 32312 \\
\hline Porophyllum lineare DC. & Ter & C.S. & 30065 \\
\hline Porophyllum ruderale (Jacq.) Cass. ${ }^{1}$ & Ter & C.S. & 30362 \\
\hline Pterocaulon alopecuroides (Lam.) DC. ${ }^{1}$ & $\mathrm{Hem}$ & C.S. & 30035 \\
\hline Pterocaulon virgatum $(\mathrm{L}$.$) DC. { }^{1}$ & Hem & $\mathrm{ND}$ & 28057 \\
\hline Senecio trixoides Gardner* & Ter & C.S. & 7128 \\
\hline Stevia lundiana DC. & Ter & C.S. & 28147 \\
\hline Tagetes minuta $\mathrm{L}^{1}{ }^{1}$ & Ter & C.S. & 7460 \\
\hline Tridax procumbens $\mathrm{L} .{ }^{1}$ & Ter & C.S. & 28950 \\
\hline Vernonia bardanoides Less. & Hem & C.S. & 30337 \\
\hline Vernonia brasiliana (L.) Druce* & $\mathrm{Hem}$ & C.S. & 4174 \\
\hline Vernonia cognata Less. $* 1,2$ & Hem & C.S. & 27701 \\
\hline Vernonia ferruginea Less. $* 1,2$ & Cam & C.S. & 7463 \\
\hline Vernonia grandiflora Less. & $\mathrm{Hem}$ & C.S. & 29363 \\
\hline Vernonia oxylepis Sch. Bip. ex Baker & Hem & C.S. & 29386 \\
\hline Vernonia rubriramea Mart. ex DC. & Cam & C.S. & 28506 \\
\hline Vernonia sp. & Hem & C.U. & 34018 \\
\hline Viguiera nudicaulis Baker* & Hem & C.S. & 7103 \\
\hline Indeterminada 1 & Hem & C.S. & 30336 \\
\hline Indeterminada 2 & Hem & C.S. & 30349 \\
\hline \multicolumn{4}{|l|}{ BEGONIACEAE } \\
\hline Begonia cucullata Willd. & Ter & C.U. & 29698 \\
\hline \multicolumn{4}{|l|}{ BIGNONIACEAE } \\
\hline Anemopaegma glaucum Mart. ex DC.* & Hem & C.S. & 4472 \\
\hline Arrabidaea brachypoda (A. DC.) Bureau & Cam & C.S. & 30321 \\
\hline Arrabidaea pulchra (Cham.) Sandwith* & Cam & C.S. & 26741 \\
\hline Jacaranda caroba (Vell.) A. DC. & Cam & C.S. & 28517 \\
\hline Jacaranda decurrens Cham. & Cam & C.S. & 29392 \\
\hline Jacaranda rufa Manso* & Cam & C.S. & 5563 \\
\hline Tabebuia ochracea (Cham.) Standley & Fan & C.S. & 29167 \\
\hline Tabebuia sp.* & Fan & C.S. & 26995 \\
\hline Zeyheria montana Mart. & Fan & C.S. & 28063 \\
\hline \multicolumn{4}{|l|}{ BIXACEAE } \\
\hline Cochlospermum regium (Schrank.) Pilger & Cam & C.S. & 28562 \\
\hline \multicolumn{4}{|l|}{ BOMBACACEAE } \\
\hline Eriotheca gracilipes (K. Schum.) A. Robins & Fan & C.S. & 28512 \\
\hline \multicolumn{4}{|l|}{ BROMELIACEAE } \\
\hline Bromelia balansae Mez & Hem & C.S. & 30371 \\
\hline Dyckia tuberosa (Vell.) Baker* & $\mathrm{Hem}$ & C.S. & 27012 \\
\hline \multicolumn{4}{|l|}{ CAMPANULACEAE } \\
\hline Lobelia exaltata Pohl & Hem & C.U. & 28064 \\
\hline
\end{tabular}


continuação

\begin{tabular}{|c|c|c|c|}
\hline Família/Espécie & F.V. & Hábitat & $\mathrm{HRCB}$ \\
\hline \multicolumn{4}{|l|}{ CAMPANULACEAE (cont.) } \\
\hline Siphocampylus sulfureus E. Wimmer & Hem & C.U. & 30038 \\
\hline \multicolumn{4}{|l|}{ CARYOCARACEAE } \\
\hline Caryocar brasiliense Cambess. & Fan & C.S. & 30002 \\
\hline \multicolumn{4}{|l|}{ CARYOPHYLLACEAE } \\
\hline Polycarpaea corymbosa (L.) Lam. & Hem & C.S. & 28006 \\
\hline \multicolumn{4}{|l|}{ CELASTRACEAE } \\
\hline Austroplenckia populnea (Reissek) Lundell & Fan & C.S. & 28058 \\
\hline \multicolumn{4}{|l|}{ CHRYSOBALANACEAE } \\
\hline Couepia grandiflora (Mart. \& Zucc.) Benth. ex Hook. f. & Fan & C.S. & 29146 \\
\hline Licania humilis Cham. \& Schltdl. & Cam & C.S. & 28584 \\
\hline Parinari obtusifolia Hook. f. & Cam & C.S. & 28566 \\
\hline \multicolumn{4}{|l|}{ CLUSIACEAE } \\
\hline Kielmeyera coriacea (Spreng.) Mart. & Fan & C.S. & 28140 \\
\hline \multicolumn{4}{|l|}{ COMMELINACEAE } \\
\hline Commelina sp. & Hem & C.U. & 28032 \\
\hline \multicolumn{4}{|l|}{ CONVOLVULACEAE } \\
\hline Evolvulus sericeus $\mathrm{Sw} . *$ & Hem & C.S. & 8161 \\
\hline Evolvulus sp. & Hem & C.S. & 28123 \\
\hline Ipomoea procurrens Meisn. & Hem & C.S. & 30361 \\
\hline Merremia digitata (Spreng.) Hallier f. & $\mathrm{Li}$ & C.S. & 28053 \\
\hline \multicolumn{4}{|l|}{ CUCURBITACEAE } \\
\hline Melancium campestre Naud. & $\mathrm{Li}$ & C.S. & 32154 \\
\hline Perianthopodus espelina Silva Manso* & $\mathrm{Li}$ & C.S. & 5048 \\
\hline \multicolumn{4}{|l|}{ CYPERACEAE } \\
\hline Bulbostylis hirtella Nees* & Hem & C.U. & 4130 \\
\hline Bulbostylis junciformis (Kunth) C.B. Clarke* & Hem & C.U. & 4140 \\
\hline Cyperus brevifolius (Rottb.) Hassk. ${ }^{1}$ & Hem & C.U. & 30341 \\
\hline Cyperus cayennensis Link $^{1}$ & Hem & $\mathrm{ND}$ & 30357 \\
\hline Cyperus haspan $\mathrm{L} . * 1$ & Hem & C.U. & 4138 \\
\hline Cyperus laetus J. Presl. \& C. Presl. ${ }^{1}$ & Geo & C.U. & 30044 \\
\hline Cyperus sesquiflorus (Torr.) Mattf. \& Kük.*1,2 & Hem & C.U. & 4161 \\
\hline Cyperus virens Michx.*1 & Hem & C.U. & 3844 \\
\hline Cyperus sp. & Hem & C.S. & 29731 \\
\hline Eleocharis minima Kunth* & Hem & C.U. & 8194 \\
\hline Fimbristylis autumnalis (L.) Roem. \& Schult. ${ }^{1,2}$ & Hem & C.U. & 29986 \\
\hline Kyllinga odorata Vahl*1 & Geo & C.U. & 4133 \\
\hline Rhyncospora albiceps Kunth & Hem & C.U. & 30341 \\
\hline Rhyncospora canescens (Maury) H. Pfeiffer & Hem & C.U. & 29422 \\
\hline Rhyncospora corimbosa (L.) Britton ${ }^{1,2}$ & Hem & C.U. & 29419 \\
\hline Rhyncospora globosa (Kunth) Roem. \& Schult. & Hem & C.U. & 28030 \\
\hline Rhyncospora loefgrenii Boeck. & Hem & C.U. & 28560 \\
\hline Rhyncospora rugosa (Vahl) S. Galé & Hem & C.U. & 30017 \\
\hline Rhyncospora sp. 1 & Hem & C.U. & 29416 \\
\hline Rhyncospora sp. 2 & Hem & C.U. & 30015 \\
\hline Scleria hirtella Boeck.* & Geo & C.U. & 4124 \\
\hline Indeterminada $1^{*}$ & Hem & C.S. & 4150 \\
\hline Indeterminada 2 & Hem & C.U. & 28943 \\
\hline \multicolumn{4}{|l|}{ DILLENIACEAE } \\
\hline Davilla elliptica A. St.-Hil. & Fan & C.S. & 28130 \\
\hline
\end{tabular}


continuação

\begin{tabular}{|c|c|c|c|}
\hline Família/Espécie & F.V. & Hábitat & $\mathrm{HRCB}$ \\
\hline \multicolumn{4}{|l|}{ DILLENIACEAE (cont.) } \\
\hline Davilla rugosa Poir. & Cam & C.S. & 28548 \\
\hline \multicolumn{4}{|l|}{ DROSERACEAE } \\
\hline Drosera communis A. St.-Hil. & Ter & C.U. & 38603 \\
\hline \multicolumn{4}{|l|}{ EBENACEAE } \\
\hline Diospyros hispida A. DC. & Fan & C.S. & 28066 \\
\hline \multicolumn{4}{|l|}{ ERIOCAULACEAE } \\
\hline Eriocaulon sp. & Hem & C.U. & 34020 \\
\hline Paepalanthus polyanthus Bong. & Hem & C.U. & 28065 \\
\hline Paepalanthus speciosus (Bong.) Koern. & Hem & C.U. & 28951 \\
\hline Paepalanthus sp. & Hem & C.U. & 29417 \\
\hline Syngonanthus caulescens (Kunth) Ruhland* & Hem & C.U. & 27724 \\
\hline Syngonanthus gracilis (Bong.) Ruhland & Hem & C.U. & 28591 \\
\hline Syngonanthus xeranthemoides (Bong.) Ruhland* & Hem & C.U. & 24661 \\
\hline Syngonanthus sp. & Hem & C.U. & 29988 \\
\hline \multicolumn{4}{|l|}{ ERYTHROXYLACEAE } \\
\hline Erythroxylum campestre A. St.-Hil. & Fan & C.S. & 28163 \\
\hline Erythroxylum cuneifolium (Mart.) O.E. Schulz* & Fan & C.S. & 3473 \\
\hline Erythroxylum suberosum A. St.-Hil. & Fan & C.S. & 29361 \\
\hline \multicolumn{4}{|l|}{ EUPHORBIACEAE } \\
\hline Croton glandulosus (L.) Müll. Arg. ${ }^{1,2}$ & Ter & C.S. & 28010 \\
\hline Croton pohlianus Müll. Arg. & Hem & C.S. & 29367 \\
\hline Euphorbia brasiliensis Lam. ${ }^{1}$ & Ter & C.S. & 30366 \\
\hline Manihot tripartita (Spreng.) Müll. Arg., ${ }^{1,2}$ & Cam & C.S. & 30004 \\
\hline Phyllanthus orbiculatus Rich. & Ter & C.S. & 28515 \\
\hline Sapium marginatum Müll. Arg. & Fan & C.S. & 30025 \\
\hline Sebastiania serrulata (Mart.) Müll. Arg. & Ter & C.S. & 28007 \\
\hline \multicolumn{4}{|l|}{ GENTIANACEAE } \\
\hline Curtia tenuifolia (Aubl.) Knobl.* & Ter & C.U. & 5596 \\
\hline Irlbachia alata (Aubl.) Maas & Hem & C.U. & 30075 \\
\hline Irlbachia oblongifolia (Mart.) Maas & Hem & C.U. & 28052 \\
\hline Schultesia gracilis Mart. & Hem & C.U. & 28046 \\
\hline \multicolumn{4}{|l|}{ GESNERIACEAE } \\
\hline Sinningia elatior (Kunth) Chautems & Hem & C.U. & 29766 \\
\hline \multicolumn{4}{|l|}{ HIPPOCRATEACEAE } \\
\hline Peritassa campestris (Cambess.) A.C. Smith & Fan & C.S. & 28958 \\
\hline Salacia crassifolia (Mart.) G. Don.* & Fan & C.S. & 4445 \\
\hline Tontelea brachypoda Miers. & Fan & C.S. & 29144 \\
\hline Tontelea micrantha (Mart. ex Schult.) A.C. Smith & Fan & C.S. & 30048 \\
\hline \multicolumn{4}{|l|}{ IRIDACEAE } \\
\hline Sisyrinchium luzula Klotzch. ex Klatt & Geo & C.S. & 29390 \\
\hline Sisyrinchium vaginatum Spreng. & Geo & C.S. & 30333 \\
\hline \multicolumn{4}{|l|}{ JUNCACEAE } \\
\hline Juncus densiflorus Kunth*1,2 & Geo & C.U. & 4144 \\
\hline \multicolumn{4}{|l|}{ LAMIACEAE } \\
\hline Eriope crassipes Benth. & Hem & C.S. & 28008 \\
\hline Hyptis caespitosa A. St.-Hil. ex Benth. & Hem & C.S. & 28020 \\
\hline Hyptis lacunosa Pohl. ex Benth. & Hem & C.S. & 29426 \\
\hline Hyptis macrantha A. St.-Hil. ex Benth.* & Hem & C.S. & 27721 \\
\hline Hyptis pulchella Briq. & Hem & C.U. & 29380 \\
\hline Hyptis suaveolens (L.) Poit..,2 & Hem & C.S. & 28037 \\
\hline
\end{tabular}


continuação

\begin{tabular}{|c|c|c|c|}
\hline Família/Espécie & F.V. & Hábitat & $\mathrm{HRCB}$ \\
\hline \multicolumn{4}{|l|}{ LAMIACEAE (cont.) } \\
\hline Hyptis virgata Benth.* & Hem & C.S. & 27334 \\
\hline Peltodon tomentosus Pohl & Hem & C.S./C.U. & 29395 \\
\hline Peltodon sp. & Hem & C.S. & 29175 \\
\hline \multicolumn{4}{|l|}{ LEGUMINOSAE-CAESALPINIOIDEAE } \\
\hline Chamaecrista cathartica (Mart.) H.S. Irwin \& Barneby & Cam & C.S. & 28559 \\
\hline Chamaecrista desvauxii (Collad.) Killip. & Cam & C.S. & 29998 \\
\hline Chamaecristaflexuosa (L.) Greene $\mathrm{e}^{1,2}$ & Cam & C.S. & 30054 \\
\hline Chamaecrista ramosa (Vogel) H.S. Irwin \& Barneby & Cam & C.S. & 28005 \\
\hline Chamaecrista rotundifolia (Pers.) Greene $\mathrm{e}^{1,2}$ & Ter & C.S. & 28014 \\
\hline Dimorphandra mollis Benth.*1,2 & Fan & C.S. & 5646 \\
\hline Hymenaea stigonocarpa Mart. ex Hayne & Fan & C.S. & 38602 \\
\hline Senna occidentalis (L.) Link ${ }^{1}$ & Ter & C.S. & 30047 \\
\hline Senna rugosa (Don) H.S. Irwin \& Barneby & Fan & C.S. & 28039 \\
\hline \multicolumn{4}{|l|}{ LEGUMINOSAE-FABOIDEAE } \\
\hline Acosmium subelegans (Mohlenbr.) Yakovlev & Fan & C.S. & 29741 \\
\hline Andira humilis Mart. ex Benth. & Cam & C.S. & 29382 \\
\hline Crotalaria pallida Ait. ${ }^{1,2}$ & Hem & C.S. & 4163 \\
\hline Crotalaria unifoliolata Benth. & Hem & C.S. & 29997 \\
\hline Eriosema sp. 1 & Hem & C.S. & 28576 \\
\hline Eriosema sp. 2 & Hem & C.S. & 28129 \\
\hline Galactia decumbens (Benth.) Hoehne & Hem & C.S. & 29729 \\
\hline Indigofera bongardiana (Kuntze) Burkart* & Hem & C.S. & 4489 \\
\hline Lupinus crotalarioides Mart. ex Benth. & Hem & C.S. & 28061 \\
\hline Machaerium acutifolium Vogel & Fan & C.S. & 28535 \\
\hline Macroptilium sp. & $\mathrm{Li}$ & C.S. & 28514 \\
\hline Stylosanthes bracteata Vogel & Ter & C.S. & 28124 \\
\hline Stylosanthes gracilis Kunth* & Ter & C.S. & 26966 \\
\hline Stylosanthes guianensis (Aubl.) Sw. ${ }^{1,2}$ & Ter & C.S. & 28529 \\
\hline Stylosanthes sp. & Ter & $\mathrm{ND}$ & 29172 \\
\hline Tephrosia rufescens Benth.* & Hem & C.S. & 4171 \\
\hline Vigna sp. & Hem & C.S. & 28009 \\
\hline Zornia glabra Desv. & Hem & C.S./C.U. & 28525 \\
\hline Zornia latifolia $\mathrm{Sm} .^{1}$ & Hem & C.S./C.U. & 34021 \\
\hline Zornia reticulata $\mathrm{Sm}$. & Hem & C.S./C.U. & 30020 \\
\hline Indeterminada 1 & $\mathrm{Li}$ & C.S. & 30338 \\
\hline Indeterminada 2 & $\mathrm{Li}$ & C.S. & 30363 \\
\hline \multicolumn{4}{|l|}{ LEGUMINOSAE-MIMOSOIDEAE } \\
\hline Stryphnodendron cf. adstringens (Mart.) Coville & Fan & C.S. & 35623 \\
\hline Stryphnodendron cf. obovatum Benth. & Fan & C.S. & 35153 \\
\hline Stryphnodendron polyphyllum Mart. & Fan & C.S. & 28578 \\
\hline \multicolumn{4}{|l|}{ LENTIBULARIACEAE } \\
\hline Genlisea filiformis A. St.-Hil.* & Hem & C.U. & 5642 \\
\hline Utricularia cucullata A. St-Hil. \& Girard & Hem & C.U. & 30375 \\
\hline Utricularia gibba L. ${ }^{1}$ & Hem & C.U. & 29671 \\
\hline Utricularia hispida Lam. & Hem & C.U. & $* *$ \\
\hline Utricularia nervosa G. Weber ex Benj. & Hem & C.U. & $* *$ \\
\hline Utricularia subulata $\mathrm{L}$. & Hem & C.U. & $* *$ \\
\hline Utricularia tricolor A. St-Hil. & Hem & C.U. & $* *$ \\
\hline Utricularia tricophylla Spruce ex Oliver & Hem & C.U. & 30542 \\
\hline Utricularia $\mathrm{sp}$ & Hem & C.U. & 30376 \\
\hline \multicolumn{4}{|l|}{ LOGANIACEAE } \\
\hline Strichnos pseudoquina A. St.-Hil. & Fan & C.S. & 28056 \\
\hline
\end{tabular}


continuação

\begin{tabular}{|c|c|c|c|}
\hline Família/Espécie & F.V. & Hábitat & $\mathrm{HRCB}$ \\
\hline \multicolumn{4}{|l|}{ LYCOPODIACEAE } \\
\hline Lycopodiella alopecuroides (L.) Cranfill & Geo & C.U. & 29768 \\
\hline Lycopodiella cernua (L.) Pic.-Serm. & Geo & C.U. & 28944 \\
\hline \multicolumn{4}{|l|}{ LYTHRACEAE } \\
\hline Cuphea micrantha Kunth & Ter & C.S. & 32315 \\
\hline Cuphea thymoides Cham. \& Schltdl. & Ter & C.S./C.U. & 28016 \\
\hline \multicolumn{4}{|l|}{ MALPIGHIACEAE } \\
\hline Banisteriopsis stellaris (Griseb.) B. Gates & Cam & C.S. & 28049 \\
\hline Byrsonima coccolobifolia Kunth & Fan & C.S. & 28055 \\
\hline Byrsonima intermedia A. Juss. ${ }^{1,2}$ & Fan & C.S. & 28011 \\
\hline Byrsonima verbascifolia (L.) Rich. ex Juss. & Fan & C.S. & 28957 \\
\hline Camarea affinis A. St-Hil. & Hem & C.S. & 29999 \\
\hline Peixotoa reticulata Griseb. & Cam & C.S. & 28159 \\
\hline \multicolumn{4}{|l|}{ MALVACEAE } \\
\hline Peltaea polymorpha (A. St.-Hil.) Krapov. \& Cristóbal & Hem & C.S. & 29409 \\
\hline Sida glaziovii K. Schum. ${ }^{1}$ & Cam & $\mathrm{ND}$ & 30342 \\
\hline Sida linifolia Cav. ${ }^{1}$ & Ter & C.S. & 29730 \\
\hline Sida spinosa L. $^{1}$ & Ter & C.S. & 29393 \\
\hline \multicolumn{4}{|l|}{ MAYACACEAE } \\
\hline Mayaca sellowiana Kunth & Hem & C.U. & 30368 \\
\hline \multicolumn{4}{|l|}{ MELASTOMATACEAE } \\
\hline Acisanthera alsinaefolia (DC.) Triana & Hem & C.U. & 30328 \\
\hline Acisanthera sp. & Hem & C.U. & 28021 \\
\hline Cambessedesia hilariana (Kunth) DC. & Cam & C.S. & 28507 \\
\hline Macairea radula (Bonpl.) DC. & Hem & C.U. & 30074 \\
\hline Miconia albicans (Sw.) Triana & Fan & C.S. & 28045 \\
\hline Miconia chamissois Naudin & Fan & C.S. & 28054 \\
\hline Miconia fallax DC. & Fan & C.S. & 28526 \\
\hline Miconia minutiflora (Bonpl.) DC. & Cam & C.S. & 28534 \\
\hline Miconia theaezans (Bonpl.) Cogn.* & Cam & C.U. & 4428 \\
\hline Microlepis oleaefolia (DC.) Triana & Hem & C.U. & 28031 \\
\hline Microlicia polystemma Naudin & Cam & C.S. & 28162 \\
\hline Rhyncanthera ursina Naudin & Hem & C.U. & 28012 \\
\hline Tibouchina gracilis (Bonpl.) Cogn. & Hem & C.U. & 28028 \\
\hline Tibouchina stenocarpa (DC.) Cogn. & Fan & C.S. & 30346 \\
\hline \multicolumn{4}{|l|}{ MYRSINACEAE } \\
\hline Rapanea guianensis Aubl.* & Fan & C.S. & 5814 \\
\hline \multicolumn{4}{|l|}{ MYRTACEAE } \\
\hline Campomanesia pubescens (DC.) O. Berg. & Fan & C.S. & 29430 \\
\hline Campomanesia sp. & Fan & C.S. & 32141 \\
\hline Eugenia bimarginata DC. & Fan & C.S. & 28513 \\
\hline Eugenia klotzchiana O. Berg.* & Cam & C.S. & 4522 \\
\hline Eugenia punicifolia (Kunth) DC. & Cam & C.S. & 28563 \\
\hline Eugenia pyriformis Cambess. & Cam & C.S. & 29429 \\
\hline Myrcia guianensis (Aubl.) DC. & Fan & C.S. & 29166 \\
\hline Psidium aerugineum $\mathrm{O}$. Berg.* & Cam & C.S. & 26479 \\
\hline Psidium australe Cambess.* & Cam & C.S. & 26476 \\
\hline Psidium cinereum Mart. ex DC. & $\mathrm{Cam}$ & C.S. & 29365 \\
\hline Psidium laruotteanum Cambess.* & Cam & C.S. & 26486 \\
\hline \multicolumn{4}{|l|}{ NYCTAGINACEAE } \\
\hline Guapira noxia (Netto) Lundell & Fan & C.S. & 29428 \\
\hline
\end{tabular}


continuação

\begin{tabular}{|c|c|c|c|}
\hline Família/Espécie & F.V. & Hábitat & HRCB \\
\hline \multicolumn{4}{|l|}{ OCHNACEAE } \\
\hline Ouratea spectabilis (Mart.) Engl. & Cam & C.S. & 29151 \\
\hline Sauvagesia erecta $\mathrm{L}$. & Ter & C.U. & 28018 \\
\hline Sauvagesia racemosa A. St.-Hil. & Hem & C.U. & 28051 \\
\hline \multicolumn{4}{|l|}{ ONAGRACEAE } \\
\hline Ludwigia nervosa (Poir.) Hara & Hem & C.U. & 28034 \\
\hline Ludwigia octovalvis (Jacq.) Raven ${ }^{1}$ & Ter & C.U. & 28029 \\
\hline Ludwigia sericea (Cambess.) Hara ${ }^{1}$ & Ter & C.U. & 3842 \\
\hline \multicolumn{4}{|l|}{ ORCHIDACEAE } \\
\hline Habenaria glazioviana Kraenzl. & Ter & C.U. & 28044 \\
\hline Habenaria obtusa Lindl. & Ter & C.U. & 32150 \\
\hline Habenaria parviflora Lindl.* & Ter & C.U. & 3852 \\
\hline Habenaria platydactyla Kraenzl.* & Ter & C.U. & 27705 \\
\hline \multicolumn{4}{|l|}{ POACEAE } \\
\hline Andropogon bicornis L.1,2 & Hem & C.U. & 34023 \\
\hline Andropogon lateralis Nees & Hem & C.U. & 30340 \\
\hline Andropogon leucostachyus Kunth ${ }^{1,2}$ & Hem & C.U. & 29732 \\
\hline Andropogon selloanus (Hack.) Hack. & Hem & C.U. & 30057 \\
\hline Aristida jubata (Arechav.) Herter & Hem & C.S. & 29427 \\
\hline Arundinella sp.* & Hem & C.S. & 4155 \\
\hline Axonopus brasiliensis (Spreng.) Kuhlm. & Hem & C.U. & 28516 \\
\hline Axonopus marginatus (Trin.) Chase & Hem & $\mathrm{ND}$ & 30332 \\
\hline Axonopus pressus (Nees ex Steud.) Parodi & Hem & C.S. & 30324 \\
\hline Axonopus siccus (Nees) Kuhlm.* & Hem & C.U. & 4149 \\
\hline Brachiaria decumbens Stapf. ${ }^{1}$ & Hem & C.S. & 4160 \\
\hline Echinolaena inflexa (Poir.) Chase $\mathrm{e}^{1,2}$ & Hem & C.S. & 1030 \\
\hline Elionurus muticus (Spreng.) Kuntze* & Hem & C.S. & 5917 \\
\hline Eragrostis leucosticta Nees ex Doell & Ter & C.S. & 30061 \\
\hline Eragrostis maypurensis (Kunth.) Steud.* & Ter & C.S. & 8173 \\
\hline Eragrostis rufescens Schrad. ex Schult. & Ter & C.S. & 29176 \\
\hline Eragrostis solida Nees & Hem & C.S. & 28575 \\
\hline Eriochrysis cayennensis Beauv. & Hem & C.U. & 28590 \\
\hline Gymnopogon foliosus (Willd.) Nees & Hem & C.S. & 28017 \\
\hline Hypogynium virgatum (Desv.) Dandy & Hem & C.U. & 28589 \\
\hline Leptocoryphium lanatum (Kunth) Nees* & Hem & C.S. & 4173 \\
\hline Loudetiopsis chrysothrix (Nees) Conert & Hem & C.S. & 28533 \\
\hline Panicum cervicatum Chase & Hem & C.S. & 30359 \\
\hline Panicum cyanescens Nees & Hem & C.S. & 30358 \\
\hline Panicum maximum Jacq. ${ }^{1}$ & Hem & C.S. & 4172 \\
\hline Panicum olyroides Kunth & Hem & C.S. & 30323 \\
\hline Paspalum gardnerianum Nees & Hem & C.S. & 29764 \\
\hline Paspalum pectinatum Nees & Hem & C.U. & 30043 \\
\hline Rhynchelytrum repens (Willd.) C.E. Hubb. ${ }^{1}$ & Ter & C.S. & 29751 \\
\hline Schizachyrium condensatum (Kunth) Nees ${ }^{1}$ & Hem & C.U. & 28154 \\
\hline Schizachyrium sanguineum (Retz.) Alston & Hem & C.S. & 28149 \\
\hline Sporobolus acuminatus (Trin.) Hack.* & Ter & C.S. & 4882 \\
\hline Steinchisma decipiens (Nees ex Trin.) W.V. Br. & Hem & C.U. & 29987 \\
\hline Trachypogon vestitus Anders. & Hem & C.U. & 30056 \\
\hline Tristachya leiostachya Nees & Hem & C.S. & 28155 \\
\hline \multicolumn{4}{|l|}{ POLYGALACEAE } \\
\hline Monina tristaniana A. St.-Hil. \& Moq.* & Hem & C.S. & 3831 \\
\hline
\end{tabular}


continuação

\begin{tabular}{|c|c|c|c|}
\hline Família/Espécie & F.V. & Hábitat & HRCB \\
\hline \multicolumn{4}{|l|}{ POLYGALACEAE (cont.) } \\
\hline Polygala angulata DC. & Ter & C.S. & 33063 \\
\hline Polygala cuspidata DC. & Hem & C.U. & 28125 \\
\hline Polygala hebeclada DC. & Ter & C.S. & 28146 \\
\hline Polygala hygrophila Kunth & Ter & C.U. & 28518 \\
\hline Polygala longicaulis Kunth & Ter & C.U. & 28555 \\
\hline Polygala tenuis DC. & Ter & C.U. & 29375 \\
\hline Polygala violacea Vahl..$^{1,2}$ & Hem & C.S. & 30367 \\
\hline \multicolumn{4}{|l|}{ PONTEDERIACEAE } \\
\hline Pontederia cf. cordata L. ${ }^{1,2}$ & Geo & C.U. & 29700 \\
\hline \multicolumn{4}{|l|}{ PORTULACACEAE } \\
\hline Portulaca mucronata Link & Hem & C.S. & 30355 \\
\hline \multicolumn{4}{|l|}{ PTERIDACEAE } \\
\hline Doryopteris lomariacea Klotzch & Geo & C.U. & 28945 \\
\hline \multicolumn{4}{|l|}{ RAFLESIACEAE } \\
\hline Pilostyles sp.* & $\operatorname{Pr}$ & C.S. & 5905 \\
\hline \multicolumn{4}{|l|}{ RAPATEACEAE } \\
\hline Cephalostemon riedelianus Koern. & Geo & C.U. & 30072 \\
\hline \multicolumn{4}{|l|}{ RHAMNACEAE } \\
\hline Crumenaria polygaloides Reissek & Hem & C.S. & 29737 \\
\hline \multicolumn{4}{|l|}{ RUBIACEAE } \\
\hline Alibertia sessilis (Vell.) K. Schum. & Cam & C.S. & 28954 \\
\hline Borreria capitata (Ruiz \& Pav.) DC., & Ter & C.S./C.U. & 28572 \\
\hline Borreria cf. poaya (A. St.-Hil.) DC. & Ter & C.S. & 30011 \\
\hline Borreria sp. 1 & Ter & C.U. & 28023 \\
\hline Borreria sp. 2 & Ter & C.U. & 30081 \\
\hline Coccocypselum lanceolatum (Ruiz \& Pav.) Pers.* & Hem & C.U. & 8190 \\
\hline Declieuxia fruticosa (Willd. ex Roem. \& Schult.) Kuntze & $\mathrm{Hem}$ & C.S. & 28135 \\
\hline Galianthe eupatorioides (Cham. \& Schltdl.) Cabral & $\mathrm{Hem}$ & C.S. & 28059 \\
\hline Palicourea rigida Kunth & Cam & C.S. & 29748 \\
\hline Richardia cf. pedicellata (K. Schum.) Kuntze ${ }^{1}$ & Ter & C.S. & 29425 \\
\hline Tocoyena brasiliensis Mart. & Fan & C.S. & 29434 \\
\hline Tocoyena formosa (Cham. \& Schltdl.) K. Schum. & Fan & C.S. & 35149 \\
\hline \multicolumn{4}{|l|}{ SAPINDACEAE } \\
\hline Serjania communis Cambess. & $\mathrm{Li}$ & C.S. & 29174 \\
\hline Talisia angustifolia Radlk. & Cam & C.S. & 29387 \\
\hline \multicolumn{4}{|l|}{ SAPOTACEAE } \\
\hline Pouteria subcaerulea Pierre ex Dubard & Cam & C.S. & 28040 \\
\hline Pouteria torta (Mart.) Radlk. & Fan & C.S. & 28953 \\
\hline Pradosia brevipes (Pierre) Pennington* & Cam & C.S. & 24576 \\
\hline \multicolumn{4}{|l|}{ SCROPHULARIACEAE } \\
\hline Buchnera juncea Cham. \& Schltdl. & Hem & C.U. & 28568 \\
\hline Buchnera lavandulacea Cham. \& Schltdl. & Hem & C.S./C.U. & 28128 \\
\hline Buchnera longifolia Kunth & Hem & C.U. & 28033 \\
\hline Buchnera ternifolia Kunth & Hem & C.U. & 28036 \\
\hline Conobea scoparioides (Cham. \& Schltdl.) Benth. & Hem & C.U. & 28582 \\
\hline Scoparia dulcis L. $^{1}$ & Hem & C.S. & 29372 \\
\hline \multicolumn{4}{|l|}{ SMILACACEAE } \\
\hline Smilax polyantha Griseb. & $\mathrm{Li}$ & C.S. & 29142 \\
\hline \multicolumn{4}{|l|}{ SOLANACEAE } \\
\hline Schwenkia hirta Klotzsch. & Ter & C.U. & 28035 \\
\hline
\end{tabular}


continuação

\begin{tabular}{|c|c|c|c|}
\hline Família/Espécie & F.V. & Hábitat & HRCB \\
\hline \multicolumn{4}{|l|}{ SOLANACEAE (cont.) } \\
\hline Schwenkia hirta Klotzsch. & Ter & C.U. & 28035 \\
\hline Solanum lycocarpum A. St.-Hil. ${ }^{2}$ & Fan & C.S. & 28166 \\
\hline \multicolumn{4}{|l|}{ STERCULIACEAE } \\
\hline Byttneria palustris Cristobal & Hem & C.U. & 29667 \\
\hline Waltheria cf. indica L. $^{2}$ & Hem & C.S. & 29415 \\
\hline Waltheria cf. polyantha K. Schum. & Hem & C.S. & 29385 \\
\hline \multicolumn{4}{|l|}{ STYRACACEAE } \\
\hline Styrax camporum Pohl & Fan & C.S. & 30546 \\
\hline \multicolumn{4}{|l|}{ SYMPLOCACEAE } \\
\hline Symplocos lanceolata (Mart.) A. DC. & Fan & C.S. & 29168 \\
\hline \multicolumn{4}{|l|}{ TURNERACEAE } \\
\hline Piriqueta rosea (Cambess.) Urb.* & Hem & C.S. & 8181 \\
\hline \multicolumn{4}{|l|}{ VERBENACEAE } \\
\hline Lippia cf. asperrima Cham.* & Hem & C.S. & 4164 \\
\hline Lippia florida Cham. & Hem & C.S. & 28153 \\
\hline Lippia lupulina Cham. & Hem & C.S. & 28136 \\
\hline Lippia pohliana Schauer & Hem & C.S. & 30053 \\
\hline Lippia cf. salviaefolia Cham. & Cam & C.S. & 30329 \\
\hline Stachytarpheta cayennensis (Rich.) Vahl ${ }^{1,2}$ & Hem & C.S. & 30078 \\
\hline \multicolumn{4}{|l|}{ VITACEAE } \\
\hline Cissus erosa (L.) Rich. & Cam & C.S. & 29760 \\
\hline \multicolumn{4}{|l|}{ VOCHYSIACEAE } \\
\hline Qualea grandiflora Mart. & Fan & C.S. & 35150 \\
\hline Qualea parviflora Mart.* & Fan & C.S. & 4629 \\
\hline Vochysia cinnamomea Pohl & Fan & C.S. & 28122 \\
\hline \multicolumn{4}{|l|}{ XYRIDACEAE } \\
\hline Abolboda pulchella Humb. \& Bonpl. & Hem & C.U. & 29423 \\
\hline Xyris asperula Mart. & Hem & C.U. & 28027 \\
\hline Xyris hymenachne Mart. & Hem & C.U. & 30540 \\
\hline Xyris jupicai L.C. Rich.* & Hem & C.U. & 4166 \\
\hline Xyris laxifolia Mart. & Hem & C.U. & 30012 \\
\hline Xyris savanensis Miq. & Ter & C.U. & 28025 \\
\hline Xyris seubertii Alb. Nielson* & Hem & C.U. & 5638 \\
\hline
\end{tabular}

*Espécimes levantadas na coleção do HRCB, **Espécimes fixados em álcool; ${ }^{1}$ Espécies ruderais segundo Kissman (1997), Kissman \& Groth (1992, 1995), Lorenzi (1991) e Mendonça et al. (1998), ${ }^{2}$ Espécies ruderais nativas do Cerrado segundo Mendonça et al. (1998).

entre as duas fisionomias representaram apenas 2,6\% do total amostrado. Um número bastante reduzido de espécies $(1,3 \%)$ foi registrado apenas na zona de contato entre o campo sujo e o campo úmido e, portanto, diante da dificuldade de percepção com relação à preferência por habitats, essas espécies não foram atribuídas a qualquer uma dessas fisionomias.

No campo sujo foram registradas 265 espécies e 56 famílias. Grande parte das famílias (41\%) esteve representada por apenas uma espécie. Das famílias mais ricas (figura 3), Bignoniaceae, Euphorbiaceae e Myrtaceae estiveram representadas somente nesta fisionomia. No total, 40 famílias ocorreram com exclusividade no campo sujo.

No campo úmido verificou-se a ocorrência de 124 espécies e 36 famílias. Cyperaceae (20 espécies) e Poaceae (12), foram as famílias mais ricas (figura 3). Dentre outras de maior riqueza, Lentibulariaceae (nove espécies), Eriocaulaceae (oito) e Xyridaceae (sete), não estiveram representadas no campo sujo.

Com relação às formas de vida, no campo sujo o componente herbáceo-subarbustivo esteve representado por $78,5 \%$ das espécies (figura 4 ), enquanto o componente arbustivo-arbóreo compreendeu $21,5 \%$, o 


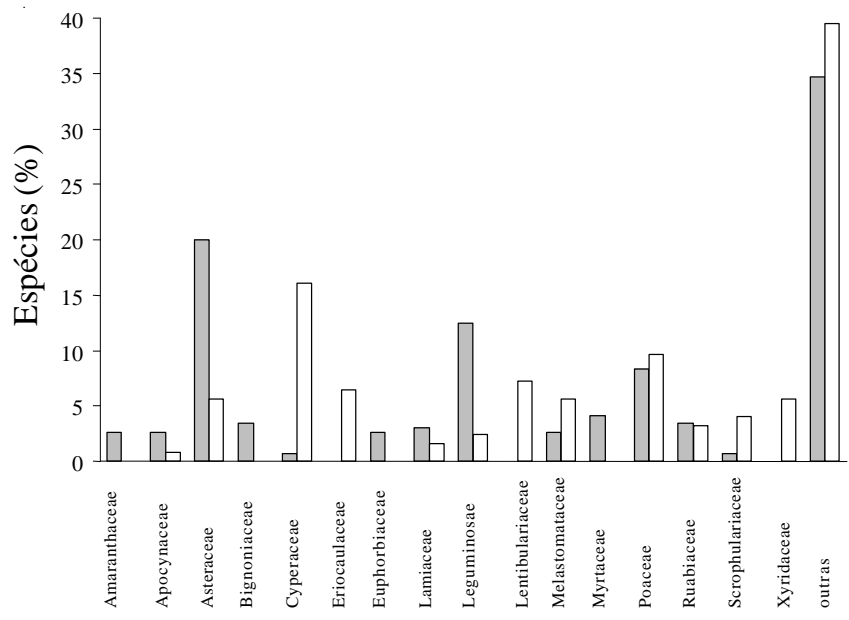

Figura 3. Distribuição em porcentagem de espécies das famílias mais ricas nas fisionomias de campo sujo (梹) e campo úmido ( $\square$ ).

Figure 3. Distribuition of species percentage of the richest families in dry grassland (媛) and swamp grassland ( $\square$ ).

que equivale a uma proporção de cerca de 3,6:1. Apocynaceae, Hippocrateaceae, Melastomataceae e Myrtaceae foram as famílias mais ricas no componente arbustivo-arbóreo do campo sujo, com quatro espécies cada.

No componente herbáceo-subarbustivo, as hemicriptófitas estiveram representadas pelo maior número de espécies entre todas as classes de formas

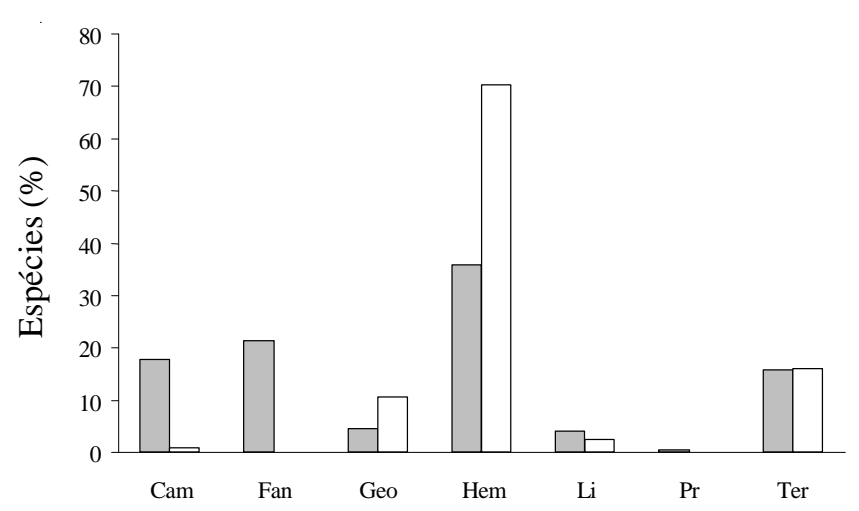

Figura 4. Distribuição em porcentagem de espécies de acordo com a forma de vida nas fisionomias de campo sujo (面) e campo úmido $(\square)$ (Cam = caméfitas; Fan = fanerófitas; Geo = geófitas $;$ Hem = hemicriptófitas $; \mathrm{Li}=$ lianas; $\operatorname{Pr}=$ parasitas; Ter $=$ terófitas).

Figure 4. Distribuition of species percentage according to life forms found in dry grassland (啹) and swamp grassland ( $\square)($ Cam $=$ chamaephytes; Fan $=$ phanerophytes; Geo = geophytes; Hem = hemicryptophytes; $\mathrm{Li}=$ vines; $\operatorname{Pr}=$ parasites; Ter $=$ terophytes $)$. de vida (figura 4), e destas as famílias mais representativas foram Asteraceae (24 espécies), Poaceae (17), Leguminosae (12), Lamiaceae (oito) e Verbenaceae (cinco). As caméfitas foram mais representadas por Asteraceae (nove espécies), Myrtaceae (sete), Bignoniaceae e Leguminosae (cinco). Entre as geófitas, prevaleceu a riqueza de Amaranthaceae com seis espécies. Na classe de lianas houve predomínio de Leguminosae (três espécies), Asclepiadaceae e Cucurbitaceae (duas). Asteraceae, com 16 espécies, Poaceae (cinco) e Euphorbiaceae (três) foram as mais representativas entre as terófitas.

No campo úmido, o único componente florístico verificado foi o herbáceo-subarbustivo, com predomínio de hemicriptófitas (figura 4), cujas famílias mais ricas foram Cyperaceae (17 espécies), Poaceae (12), Lentibulariaceae (nove), Eriocaulaceae (oito), Melastomataceae e Xyridaceae (seis). Dentre as outras classes de formas de vida, as geófitas estiveram mais representadas por Cyperaceae (três espécies), Araceae e Lycopodiaceae (duas); as terófitas por Orchidaceae (quatro), Polygalaceae e Rubiaceae (três); as lianas por Apocynaceae, Asclepiadaceae e Asteraceae com apenas uma espécie cada. As caméfitas estiveram representadas apenas por Miconia theaezans (Melastomataceae).

\section{Discussão}

Os resultados obtidos demonstram a existência de uma considerável riqueza florística para a área estudada, aproximadamente $18 \%$ do total de espécies compilado por Mendonça et al. (1998) para a flora campestre do bioma Cerrado. Embora o objetivo do presente estudo não tenha sido o de analisar os efeitos da influência antrópica sobre a composição florística da área em questão, o fato dessa área estar sujeita a tal influência nos remete a questões cujas respostas são matérias controversas, tais como: Essas influências externas estariam afetando a riqueza de espécies encontrada? De que forma?

Ainda que não existam dados relativos à intensidade da intervenção humana na área, principalmente no que se refere à ação de pastejo e de queimadas, a porcentagem de espécies consideradas como ruderais $(15,6 \%)$ foi relativamente elevada, e desta forma poderia estar contribuindo para elevar a riqueza de espécies, embora cerca de $50 \%$ dessas espécies tidas como ruderais sejam próprias do bioma (Mendonça et al. 1998). Por outro lado, segundo Filgueiras (2002), as espécies invasoras podem representar sérias ameaças 
à flora do Cerrado competindo e eliminando espécies nativas. Além disso, a notável presença de pequenas manchas de Brachiaria decumbens, planta exótica e fortemente invasora com monodominância nos locais de sua ocorrência, certamente estaria desfavorecendo a diversidade da flora nativa tendo em vista que o número de espécies por área geralmente é elevado no Cerrado e casos de monodominância são raros (Filgueiras 2002).

Com relação às queimadas, seus efeitos sobre a vegetação do Cerrado dependem da freqüência, intensidade e época em que ocorrem e trazem conseqüências diretas à estrutura e à composição florística (Silva 1987, Coutinho 2002, Hoffmann \& Moreira 2002). Queimadas esporádicas favorecem a manutenção da diversidade florística, sobretudo do componente herbáceo-subarbustivo (Coutinho 2002). Por outro lado, a reincidência do fogo pode acarretar um empobrecimento da flora, que se faz sentir, principalmente, pela redução de espécies arbustivoarbóreas (Hoffmann \& Moreira 2002). Dessa forma, o fogo exerce um importante papel na manutenção das fisionomias do Cerrado, de modo que, na área do presente estudo, poderia estar favorecendo a ocorrência de fisionomias campestres.

A constatação da maior riqueza de espécies no campo sujo em relação ao campo úmido deve levar em conta dois aspectos principais: as diferenças nos tamanhos das áreas ocupadas por cada fisionomia, cerca de dois terços e um terço, respectivamente, e as características edáficas dos ambientes. O conceito de que a riqueza de espécies tem forte relação com o tamanho da área (Odum 2001) é válido, sobretudo quando se comparam comunidades afins. Como no presente caso tratam-se de comunidades distintas, estando uma delas sujeita a uma condição bastante específica de estresse ambiental ocasionado pelo alagamento sazonal do solo, as relações entre número de espécies e tamanho de área não necessariamente correspondem. Restrições impostas por solos alagados para a germinação e estabelecimento de espécies de plantas, em consequiência da deficiência de oxigênio, têm sido relatadas para diversas formações vegetais que usualmente apresentam baixa riqueza e diversidade florística (Ivanauskas et al. 1997, Scarano 1998, Araújo et al. 2002, Cattanio et al. 2002). A despeito da menor riqueza encontrada no campo úmido, salienta-se a grande contribuição de espécies provenientes dessa fisionomia para a composição florística da área como um todo.

Além das diferenças quanto à riqueza de espécies entre as fisionomias estudadas, a análise dos dados revelou que a distinção na composição florística entre elas é acentuada, tendo em vista o reduzido número de espécies compartilhadas (10 spp.) e a pouca coincidência existente entre as famílias (16), sobretudo aquelas que se destacam como mais ricas nestas fisionomias.

Famílias como Asteraceae, Poaceae, Melastomataceae, Leguminosae, Myrtaceae, Bignoniaceae, Rubiaceae, Lamiaceae, Apocynaceae e Euphorbiaceae, que se destacaram em riqueza no campo sujo, têm sido comumente apontadas entre as mais ricas em diversos estudos da flora do cerrado (s.l.) (Heringer et al. 1977, Leitão Filho 1992, Ratter et al. 1996, Felfili et al. 1998, Mendonça et al. 1998) e, excetuando-se as três primeiras, quando representadas, pouco se destacaram no campo úmido. Entre essas, Leguminosae, Rubiaceae e Apocynaceae figuram entre as mais ricas tanto no componente herbáceo-subarbustivo quanto no arbustivo-arbóreo do cerrado (s.l.) (segundo Goodland 1970, Heringer et al. 1977, Leitão Filho 1992, Mantovani \& Martins 1993, Mendonça et al. 1998). Por sua vez, Asteraceae e Poaceae encontram-se praticamente restritas ao componente herbáceo-subarbustivo e, consequentemente, no cerrado (s.l.) apresentam maior grau de riqueza principalmente nas fisionomias campestres ou savânicas (Mantovani \& Martins 1993, Batalha \& Martins 2002). Como a maior parte dos representantes dessas famílias é composta por espécies heliófilas (Coutinho 1978, Filgueiras 2002) estas encontram nas fisionomias abertas locais ideais para seu estabelecimento, a exemplo do constatado neste estudo, tanto no campo sujo quanto no campo úmido.

Em relação às famílias mais ricas no campo úmido, Cyperaceae, Lentibulariaceae, Eriocaulaceae, Xyridaceae e Scrophulariaceae usualmente são pouco representadas, quando não ausentes, nas fisionomias próprias do cerrado (s.l.), como pode ser constatado em Mantovani \& Martins (1993), Ratter et al. (1996), Batalha et al. (1997), Castro et al. (1999), Durigan et al. (1999), Batalha \& Mantovani (2000), Batalha \& Martins (2002). Muitos dos representantes dessas famílias são tidos como característicos de vegetações que se desenvolvem em terrenos alagáveis (Joly 1998), tais como "brejos" ou "várzeas" (Loefgren 1890, Filgueiras 2002), e veredas (Schiavini \& Araújo 1989, Mendonça et al. 1998, Araújo et al. 2002, Guimarães et al. 2002). Além disso, essas famílias se encontram bem representadas na flora dos campos rupestres (Giulietti et al. 1987, Giulietti \& Hensold 1990, Pirani et al. 1994), embora o número de espécies em comum com o campo úmido estudado seja reduzido.

A preferência dos táxons presentes no campo úmido por ambientes alagáveis ou com excedente hídrico 
subsuperficial parece se confirmar em outras comparações. Do total de espécies encontradas no campo úmido, 65 foram também listadas por Mendonça et al. (1998), tendo como ambiente de ocorrência, veredas, campos úmidos e brejos, além de campos rupestres. Da mesma forma, 57 dessas espécies são citadas por Araújo et al. (2002) para a flora das veredas de Uberlândia (MG). Por outro lado, poucas espécies encontradas no campo úmido (30 spp.) são eventualmente citadas para as fisionomias do cerrado (s.l.) que, caracteristicamente, se estabelecem em solos bem drenados (Mantovani \& Martins 1993, Batalha et al. 1997, Mendonça et al. 1998, Durigan et al. 1999, Batalha \& Mantovani 2000).

Vários autores (e.g., Oliveira Filho et al. 1989, Pivello et al. 1998, Uhlmann et al. 1998, Uhlmann 2003) têm relacionado as variações estruturais e florísticas, típicas dos ambientes do Cerrado, com as características de drenagem do solo, as quais são condicionadas por fatores geomórficos. Uhlmann (2003) relata que as espécies arbóreas do cerrado (s.l.) sofrem restrições quanto ao seu estabelecimento sobre solos hidromórficos, de modo que a água em subsuperfície seria um fator determinante das variações na vegetação. Os resultados obtidos neste estudo parecem concordar com os relatos de Uhlmann (2003), uma vez que, quando se comparam as formas de vida presentes nas duas fisionomias, nota-se que as espécies arbustivo-arbóreas (fanerófitas) se encontram restritas ao campo sujo, onde o solo é melhor drenado. Além disso, as caméfitas (subarbustos) são mais proeminentes nesta fisionomia, e muito pouco representadas no campo úmido (uma única espécie), sugerindo que esta forma de vida também sofre restrições impostas pelas condições de drenagem do solo. Estas restrições têm implicações diretas, não apenas sobre os aspectos fisionômicos da vegetação, mas também sobre a composição florística das fisionomias estudadas. Tais implicações podem ser evidenciadas pelo grande número de famílias (32), representadas entre as fanerófitas e caméfitas, que ocorreram com exclusividade no campo sujo.

De forma semelhante, no que diz respeito às formas herbáceas, embora tenha sido constatada a prevalência das hemicriptófitas, tanto no campo sujo quanto no campo úmido, a concordância entre os táxons, dentro de cada classe de forma de vida, nas duas fisionomias estudadas, foi muito pequena, mesmo em nível de família, como pode ser verificado na comparação entre as famílias mais ricas de acordo com a forma de vida para cada fisionomia. Exceções podem ser apontadas no caso de algumas famílias que apresentaram ampla distribuição, a exemplo de Poaceae. Esta família esteve bem representada no campo sujo e no campo úmido, mas sem registro de espécies em comum a essas fisionomias. Nesse caso as restrições impostas pelas condições de drenagem parecem atuar mais em nível específico, e a disponibilidade hídrica a pouca profundidade pode ser um fator favorável à parte de seus representantes, como sugere Lütge (1997).

Comparações entre fisionomias semelhantes, em diferentes áreas de Cerrado, têm indicado elevados níveis de heterogeneidade florística, tanto para o componente arbustivo-arbóreo (Ratter et al. 1996, Felfili \& Silva Júnior 1993) quanto para a comunidade de plantas herbáceo-subarbustivas, mesmo a curtas distâncias (Felfili \& Silva Júnior 1993, Filgueiras 2002). No presente estudo, verificou-se que a heterogeneidade de composição de espécies é muito acentuada entre as diferentes fisionomias, mesmo sendo contíguas, o que estaria contribuindo para a elevada riqueza de espécies da área. Considerando-se que esta constatação provavelmente se dê numa escala mais ampla no Cerrado, pequenas variações ambientais relacionadas a grandes variações florísticas, esse seria um dos fatores responsáveis pela grande diversidade beta desse bioma.

Agradecimentos - Os autores agradecem ao CNPq pela concessão da bolsa de mestrado ao primeiro autor; ao Sr. Mário Sanches, pela disponibilização da área para o estudo; aos especialistas que auxiliaram nas identificações, em especial: A. Furlan, H.M. Longhi-Wagner, J.N. Nakajima, J. Semir, M.A. Farinaccio, M.L. Kawasaki, P.T. Sano, R. Monteiro, R. Udulutsch, R.L. Esteves, S.L. JungMendaçolli, T.S. Filgueiras, V.A. Ditrich, V.C. Souza, V.F.O. Miranda; e aos Srs. Prof. Dr. Frederico F. Mauad e José R. Maramarque (CRHEA/EESC - USP) pelo fornecimento dos dados climáticos.

\section{Referências bibliográficas}

ARAÚJO, G.M., BARBOSA, A.A.A., ARANTES, A.A. \& AMARAL, A.F. 2002. Composição florística de veredas no Município de Uberlândia, MG. Revista Brasileira de Botânica 25:475-493.

BATALHA, M.A.,ARAGAKI, S. \& MANTOVANI, W. 1997. Florística do Cerrado de Emas (Pirassununga, SP). Boletim de Botânica da Universidade de São Paulo16:49-64.

BATALHA, M.A. \& MANTOVANI, W. 2000. Reproductive phenological patterns of Cerrado plant species at the Pé-de-Gigante reserve (Santa Rita do Passa Quatro, SP, Brazil): a comparison between the herbaceous and woody floras. Revista Brasileira de Biologia 60:129-145. 
BATALHA, M.A. \& MARTINS, F.R. 2002. The vascular flora of the cerrado in Emas National Park (Goiás, Central Brazil). Sida 20:295-311.

CASTRO, A.A.J.F., MARTINS, F.R., TAMASHIRO, J.Y. \& SHEPHERD, G.J. 1999. How rich is flora of Brazilian cerrados? Annals of Missouri Botanical Garden 86:192-224.

CATTANIO, J.H., ANDERSON, A.B. \& CARVALHO, M.S. 2002. Floristic composition and topographic variation in a tidal floodplain Forest in the Amazon Estuary. Revista Brasileira de Botânica 25:419-430.

COUTINHO, L.M. 1978. O conceito de Cerrado. Revista Brasileira de Botânica 7:17-23.

COUTINHO, L.M. 2002. O bioma do cerrado. In Eugen Warming e o cerrado brasileiro: um século depois (A.L. Klein, ed.). Editora da Unesp, São Paulo, p.77-91.

COUTO, E.G., RESENDE, M.B. \& RESENDE, S.B. 1985. Terra ardendo. Ciência Hoje 3:48-57.

CRONQUIST, A. 1988. An integrated system of classification of flowering plants. Columbia University Press, New York.

DURIGAN, G., BACIC, M.C., FRANCO, G.A.D.C. \& SIQUEIRA, M.F. 1999. Inventário florístico no cerrado da Estação Ecológica de Assis, SP. Hoehnea 26:148-172.

EITEN, G. 1963. Habitat flora of Fazenda Campininha, São Paulo, Brasil. In Simpósio sobre o Cerrado (M.G. Ferri, ed.). Editora da Universidade de São Paulo, São Paulo, p.179-231

EITEN, G. 1983. Classificação da vegetação do Brasil. CNPq, Brasília.

EITEN, G. 1992. Natural Brazilian vegetation types and their causes. Anais da Academia Brasileira de Ciências 64:35-65.

FELFILI, J.M. \& SILVAJÚNIOR, 1993. A comparative study of cerrado (sensu stricto) vegetation in Central Brazil. Journal of Tropical Ecology 9:277-289.

FELFILI, J.M., SILVAJÚNIOR., M.C., FILGUEIRAS, T.S. \& NOGUEIRA, P.E. 1998. Comparison of cerrado (sensu stricto) vegetation in central Brazil. Ciência e Cultura 50:237-243.

FILGUEIRAS, T.S. 2002. Herbaceous plant communities. In The Cerrados of Brazil: Ecology and natural history of a neotropical savanna (P.S. Oliveira \& J.R. Marquis, eds.). Columbia University Press, New York, p.121-139.

GIULIETTI, A.M., MENEZES, N.L., PIRANI, J.R., MEGURO, M. \& WANDERLEY, M.G.L. 1987. Flora da Serra do Cipó, Minas Gerais: caracterização e lista de espécies. Boletim de Botânica da Universidade de São Paulo 9:1-151.

GIULIETTI, A.M. \& HENSOLD, N. 1990. Padrões de distribuição geográfica dos gêneros de Eriocaulaceae. Acta Botanica Brasilica 4:133-158.

GOODLAND, R. 1970. Plants of cerrado vegetation of Brazil. Phytologia 20:57-78.

GUIMARÃES, A.J.M.,ARAÚJO, G.M. \& CORRÊA, G.F. 2002. Estrutura fitossociológica em área natural e antropizada de uma vereda em Uberlândia, MG. Acta Botanica Brasilica 16:317-329.
HERINGER, E.P., BARROSO, G.M., RIZZO, J.A. \& RIZZINI, C.T. 1977. A flora do cerrado. In IV Simpósio sobre cerrado: bases para a utilização agropecuária (M.G. Ferri, coord.). Itatiaia/ Edusp, Belo Horizonte/São Paulo, p.211-232.

HOFFMANN, A.W. \& MOREIRA, A.G. 2002. The role of fire in population dynamics of woody plants. In The Cerrados of Brazil: Ecology and natural history of a neotropical savanna (P.S. Oliveira \& J.R. Marquis, eds.). Columbia University Press, New York, p.159-177.

IVANAUSKAS, N.M., RODRIGUES, R.R. \& NAVE,A.G. 1997. Aspectos ecológicos de um trecho de floresta de brejo em Itatinga, SP: florística, fitossociologia e seletividade de espécies. Revista Brasileira de Botânica 20:139-154.

JOLY, A.B. 1998. Botânica: introdução à taxonomia vegetal. Edusp, São Paulo.

KISSMAN, K.G. 1997. Plantas infestantes e nocivas. v.1. Basf S.A., São Paulo.

KISSMANN, K.G. \& GROTH, D. 1992. Plantas infestantes e nocivas. v.2. Basf S.A., São Paulo.

KISSMANN, K.G. \& GROTH, D. 1995. Plantas infestantes e nocivas. v.3. Basf S.A., São Paulo.

KLINK, C.A., MOREIRA, A.G. \& SOLBRIG, O.T. 1993. Ecological impact of agricultural development in the Brazilian cerrados. In The World's savanna: economic driving forces, ecological constraints and policy options for sustainable land use (M.D. Young \& O.T. Solbrig, eds.). Man and biosphere series. Unesco/Parthenon, Paris, v.12, p.259-282.

KÖPPEN, W. 1948. Climatologia. Fondo de Cultura Económica, México.

LEITÃO FILHO, H.F. 1992. A flora arbórea dos cerrados do Estado de São Paulo. Hoehnea 19:151-163.

LOEFGREN, A. 1890. Contribuição para a botânica paulista. Região Campestre. Boletim da Comissão Geográfica e Geológica do Estado de São Paulo 5:3-51.

LORENZI, H. 1991. Plantas daninhas do Brasil. Editora Plantarum, Nova Odessa.

LÜTGE, U. 1997. Physiological ecology of tropical plants. Springer-Verlag, Berlin.

MANTOVANI, W. \& MARTINS, F.R. 1993. Florística do cerrado na Reserva Biológica de Moji-Guaçu - SP. Acta Botanica Brasilica. 7:33-59.

MENDONÇA, R.C., FELFILI, J.M., WALTER, B.M.T., SILVA JÚNIOR, M.C., REZENDE, A.V., FILGUEIRAS, T.S. \& NOGUEIRA, P.E. 1998. Flora Vascular do Cerrado. In Cerrado: ambiente e flora (S.M. Sano \& S.P. Almeida, eds.). Embrapa/CPAC, Brasília, p.289-556.

MÜELLER-DOMBOIS, D. \& ELLENBERG, H. 1974. Aims and methods of vegetation ecology. John Wiley \& Sons, New York.

ODUM, E.P. 2001. Fundamentos de ecologia. Fundação Calouste Gulbekian, Lisboa.

OLIVEIRA FILHO, A.T., SHEPHERD, G.J., MARTINS, F.R. \& STUBBLEBINE, W.H. 1989. Environmental factors affecting physiognomic and floristic variation in an area of cerrado in central Brazil. Journal of Tropical Ecology $5: 413-431$. 
PIRANI, J.R., GIULIETTI, A.M., MELLO-SILVA, R. \& MEGURO, M. 1994. Checklist and patterns of geographic distribuition of the vegetation of Serra do Ambrósio, Minas Gerais, Brazil. Revista Brasileira de Botânica 17:133-147.

PIVELLO, V.R., BARBIERI, R.F., RUGGIERO, P.G.C. \& OLIVEIRA FILHO, F. 1998. Análise da variação fisionômica na Arie Cerrado Pé-de-Gigante (Santa Rita do Passa Quatro-SP) em relação às características pedológicas locais. In IV Simpósio de Ecossistemas Brasileiros (S.Watanabe, coord.). Aciesp, São Paulo, v.3, p.7-29.

POLHILL, R.M. \& RAVEN, P.H. (eds.). 1981. Advances in legume systematics. Part 1. Royal Botanical Gardens, Kew.

PONÇANO, W.L., CARNEIRO, C.D.R., BRITICHI, C.A., ALMEIDA, F.F.M. \& PRANDINI, F.L. 1981. Mapa geomorfológico do Estado de São Paulo. v.1. Instituto de Pesquisas Tecnológicas, São Paulo.

PRADO, H. 1997. Os solos do Estado de São Paulo: mapas pedológicos. Editora Hélio do Prado, Piracicaba.

RATTER, J.A., BRIDGEWATER, S., ATKINSON, R. \& RIBEIRO, J.F. 1996. Analysis of the floristic composition of the Brazilian cerrado vegetation II: comparison of the woody vegetation of 98 areas. Edinburg Journal of Botany 53:153-180.

RATTER, J.A., RIBEIRO, J.F. \& BRIDGEWATER, S. 1997. The Brazilian cerrado vegetation and threats to its biodiversity. Annals of Botany 80:223-230.

RAUNKIAER, C. 1934. The life forms of plants and statistical geography. Clarendon, Oxford.
RIBEIRO, J.F., SANO, S.M., MACÊDO, J. \& SILVA, J.A. 1983. Os principais tipos fitofisionômicos da região dos cerrados. Boletim de Pesquisa Embrapa/CPAC 21:5-28.

RIBEIRO, J.F. \& WALTER, B.M.T. 1998. Fitofisionomias do Bioma Cerrado. In Cerrado: ambiente e flora (S.M. Sano \& S.P. Almeida, eds.). Embrapa/CPAC, Brasília, p.89-166.

RIZZINI, C.T. 1979. Tratado de Fitogeografia do Brasil. v.2. Aspectos sociológicos e florísticos. Edusp, São Paulo.

SCARANO, F.R. 1998. A comparison of dispersal, germination and establishment of woody plant subject to distinct flooding regimes in Brazilian flood-plane forests estuarine vegetation. Oecologia Brasiliensis 4:177-194.

SCHIAVINI, I. \& ARAÚJO, G.M. 1989. Considerações sobre a vegetação da Reserva Ecológica do Panga (Uberlândia). Sociedade e Natureza 1: 61-66.

SILVA, J.F. 1987. Responses of savannas to stress and disturbance: species dynamics. In Determinants of tropical savannas (B.H. Walker, ed.). IUSB, Paris, p.141-156.

TRYON, R.M. \& TRYON, A.F. 1982. Ferns and allied plants. Springer-Verlag, New York.

UHLMANN, A., GALVÃO, F. \& SILVA, S.M. 1998. Análise da estrutura de duas unidades fitofisionômicas de savana (Cerrado) no Sul do Brasil. Acta Botanica Brasilica 12:231-247.

UHLMANN, A. 2003. Análise estrutural de duas áreas de vegetação savânica (Cerrado) sob influência de gradientes ambientais complexos. Tese de doutorado, Universidade Estadual de Campinas, Campinas. 